\title{
EDITORIAL
}

\section{Treatment of severe MRSA infections: current practice and further development}

\author{
José-Artur Paiva $^{1 *}$ and Philippe Eggimann²
}

๑ 2016 Springer-Verlag Berlin Heidelberg and ESICM

Staphylococcus aureus is one of the "ESKAPE pathogens", considered to require urgent development of new therapies, in spite of some decline in the incidence of methicillin-resistant S. aureus (MRSA) infections. Vancomycin, a glycopeptide with an excellent spectrum of activity against Gram-positive pathogens through inhibition of cell wall synthesis, has been the mainstay of treatment for MRSA. However, MRSA infections are associated with increased morbidity and mortality, when compared with methicillin-sensitive $S$. aureus (MSSA), and several weaknesses have been identified related to vancomycin use, namely slower bacterial killing than oxacillin, poor penetration in the lungs and central nervous system, and frequent underdosage in critically ill patients as a result of increased volume of distribution and renal hyperfiltration [1].

Four main strategies may be used to circumvent the problems related with vancomycin use.

One is the use of individualized dosing of vancomycin to reach the PK/PD target of AUC/MIC $\geq 400$ that seems to improve clinical outcome [1], both in pneumonia and bacteremia. According to the 2009 Infectious Diseases Society of America (IDSA) vancomycin therapeutic guidelines [2], a loading dose of $25-30 \mathrm{mg} / \mathrm{kg}$ actual body weight should be used, followed by $15-20 \mathrm{mg} /$ $\mathrm{kg}$ q8-12 h. Vancomycin serum levels and vancomycin MRSA MIC by Etest must be measured routinely. Trough levels between 15 and $20 \mu \mathrm{g} / \mathrm{mL}$ are recommended [2], but the probability of target attainment is unlikely in high vancomycin MIC infections, and MIC values above $1 \mu \mathrm{g} /$ $\mathrm{mL}$ significantly predict treatment failure and mortality [3]. Although these outcomes may not reflect antibiotic

\footnotetext{
*Correspondence: jarturpaiva@gmail.com

${ }^{1}$ Department of Emergency and Intensive Care, Faculty of Medicine, Grupo de Infecçao e Sepsis, Centro Hospitalar Sao Joao, University of Porto, Porto, Portugal

Full author information is available at the end of the article
}

failure per se but may rather be a marker of specific pathogen virulence characteristic, as a similar association between high vancomycin MIC and outcomes was reported in MSSA infections treated with flucloxacillin [4], it seems wise to aim at higher vancomycin levels in the case of MRSA MIC > $1 \mu \mathrm{g} / \mathrm{mL}$. However, these higher doses are associated with increased incidence of nephrotoxicity. Continuous infusion has been associated with lower rates of nephrotoxicity (nephrotoxicity threshold around $28 \mu \mathrm{g} / \mathrm{mL}$ ), higher steady state concentration, faster achievement of target concentrations, less variability in serum concentrations, and simpler AUC assessment, compared with intermittent dosing [5]; however, there is no evidence of higher effectiveness of the continuous regimen [6].

Another proposed strategy is the use of more recently developed antibiotics with activity against MRSA. Globally, no drug has shown superiority to vancomycin in the treatment of MRSA infections with the possible exception of linezolid in hospital-acquired pneumonia (HAP). A multicenter randomized controlled trial (RCT) comparing vancomycin to linezolid in the treatment of MRSA HAP [7] showed that both clinical and microbiological cure rates were significantly higher in the linezolid arm compared with vancomycin, but 60-day mortality was similar in both arms. A recent meta-analysis included data from the above trial and observed similar efficacies for linezolid and vancomycin, including in microbiologically proven MRSA pneumonia [8]. The lipopeptide daptomycin, which is inactivated by pulmonary surfactant, is currently the only antibiotic to have shown noninferiority to vancomycin in the treatment of MRSA bacteremia and even a possible superiority in infections caused by MRSA with high vancomycin MIC [9]. Dosages of $8-10 \mathrm{mg} / \mathrm{kg} / \mathrm{day}$ should be used for complicated bacteremia and, preferably, in combination with other agents [10], both to improve outcomes and to decrease the emergence of resistance,

\section{Springer}




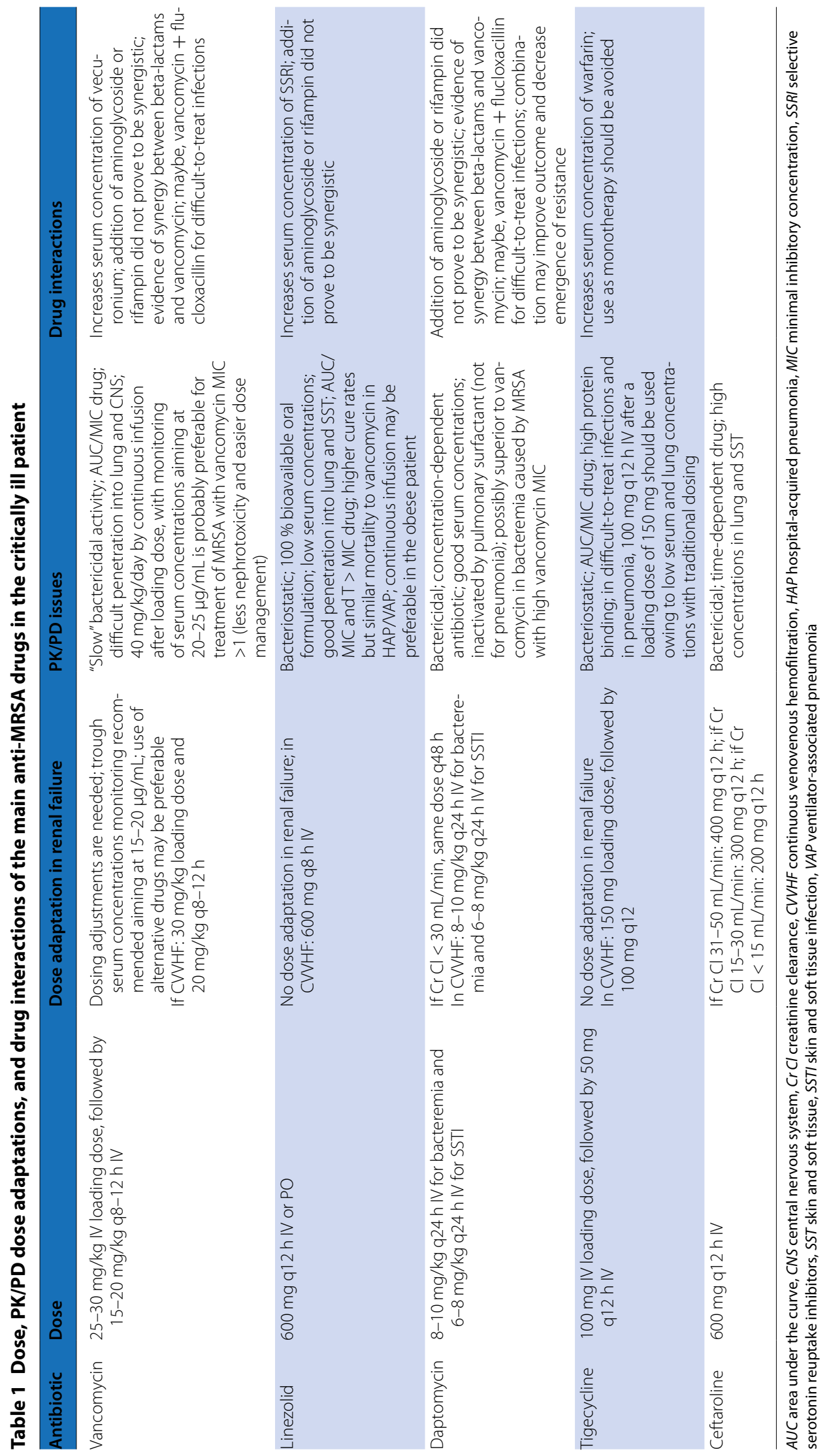


which has been described mainly associated with prior exposure to vancomycin and retained prosthetic devices. The development of ceftaroline and ceftobiprole, cephalosporins with in vitro activity against MRSA owing to their affinity for the penicillin-binding protein PBP2a, offers great promise in the treatment of MRSA, as $\beta$-lactams are associated with improved clinical outcomes when compared with glycopeptides for the treatment of MSSA infections [11]. These agents should be restricted to the treatment of MRSA infections, as it is likely that usage will be associated with increased rates of resistance that have already been observed. Tigecycline, a glycylcycline highly active against MRSA in vitro, cannot be recommended as first-line therapy in serious MRSA infections, as there is insufficient data available and reports exist of higher mortality and lower cure probably due to PK/PD considerations including high protein biding, inadequate AUC/ $\mathrm{MIC}$ with standard dosing, low serum concentrations, and poor penetration into some tissues [12]. However, it may be useful, in combination regimens, namely for skin and soft tissue or intra-abdominal infections [13]. Oritavancin, dalbavancin, and telavancin are semisynthetic lipopolypeptide analogues of vancomycin. All three show activity against MRSA and vancomycin-intermediate $S$. aureus (VISA) and oritavancin also against vancomycin-resistant S. aureus (VRSA). The long half-lives and complex PK of the first two make them unsuitable for critically ill patients unless other options do not exist [12].

Thirdly, combination therapy may be used. Combinations of two primary active agents (such as vancomycin plus linezolid) or the addition of gentamicin or rifampin to either vancomycin or daptomycin did not prove to be synergistic [6]. Rifampin, owing to its activity in biofilms, may retain a role as a secondary agent in prosthetic valve endocarditis and bone/joint infections with or without infected implants, but should only be started after clearance of bacteremia. Gentamicin's role is restricted to native valve endocarditis or as an adjunctive agent to daptomycin in bacteremia non-responsive to vancomycin. Cotrimoxazole may represent a potent alternative to all existing anti-staphylococcal agents provided that in vitro activity is demonstrated [6]. Although MRSA is inherently resistant to nearly all $\beta$-lactam antibiotics, this class of drugs has consistently shown evidence of synergy with either vancomycin or daptomycin in multiple in vitro studies and in a small number of observational studies. The mechanism may include $\beta$-lactam-induced potentiation of host defense peptide activity against $S$. aureus, $\beta$-lactam-induced alteration of MRSA cell wall which allows for improved vancomycin binding, or a "see-saw" effect whereby reduced vancomycin susceptibility results in reduced transcription of $m e c A$ and increased susceptibility to $\beta$-lactams [14]. In a retrospective cohort monocentric study of patients with MRSA bacteremia, those who received vancomycin plus one $\beta$-lactam were more likely to experience microbiological eradication of MRSA than patients who received vancomycin alone [14]. More recently, in a pilot multicenter RCT for MRSA bacteremia [15], patients received vancomycin $1.5 \mathrm{~g}$ IV twice daily and were randomly assigned to flucloxacillin 2 g IV 6 hourly for 7 days or no additional therapy. The combination therapy group showed shorter duration of MRSA bacteremia and there was no difference in 28- and 90-day mortality, metastatic infection, nephrotoxicity, or hepatotoxicity (Table 1).

Lastly, both active and passive immunotherapy against S. aureus are undergoing intense research efforts. Unfortunately, almost all attempts to provide active immunization against $S$. aureus failed and experts wonder if such an approach may result in clinical implications. Nonspecific passive immunotherapy has been reported to be useful in the control of the effect of $S$. aureus exotoxin such as Panton-Valentine leukocidin which may be responsible for very severe forms of infections. Experts currently recommend the combination of high dose nonspecific human immunoglobulins with an antibiotic able to downregulate its production such as clindamycin, rifampin, or linezolid [16]. Biotechnologies resulted in the production of highly specific human monoclonal antibodies. Those targeted at $S$. aureus toxin A, with efficacy against both MSSA and MRSA, seem very promising and their use, either as prophylaxis (MEDI4893: NCT02296320) [17] or adjunctive treatment (AR-301: NCT01589185) of S. aureus infections, is currently under investigation in multicenter phase II clinical studies.

In conclusion, in the critically ill patient, individualized dosing of vancomycin, aiming at an AUC/MIC $\geq 400$, and therefore with monitoring of serum levels and information about the Etest vancomycin MIC, is the correct strategy for vancomycin use. In MRSA HAP/VAP and in MRSA bacteremia, linezolid and daptomycin may be preferable, respectively, especially in high MIC MRSA and, in the case of daptomycin, using high dose and combination therapy. In difficult-to-treat MRSA bacteremia cases, combination of vancomycin or daptomycin with an anti-staphylococcal $\beta$-lactam may improve results in terms of microbiological eradication.

\footnotetext{
Author details

${ }^{1}$ Department of Emergency and Intensive Care, Faculty of Medicine, Grupo de Infecçao e Sepsis, Centro Hospitalar Sao Joao, University of Porto, Porto, Portugal. ${ }^{2}$ Department of Emergency and Intensive Care, Faculty of Biology and Medicine, Centre Hospitalier Universitaire Vaudois (CHUV), Lausanne, Switzerland.
}

Received: 24 August 2016 Accepted: 22 September 2016 Published online: 4 October 2016 


\section{References}

1. de Gatte MDMF, Revilla N, Calvo MV, Dominquez-Gil A, Sanchez-Navarro A (2007) Pharmacokinetic/pharmacodynamic analysis of vancomycin in ICU patients. Intensive Care Med 33:279-285

2. Rybak MJ, Lomaestro BM, Rotscahfer JC, Moellering RC et al (2009) Vancomycin therapeutic guidelines: a summary of consensus recommendations from the Infectious Diseases Society of America, the American Society of Health-System Pharmacists, and the Society of Infectious Diseases Pharmacists. Clin Infect Dis 49:325-327

3. Van Hal SJ, Lodise TP, Paterson DL (2012) The clinical significance of vancomycin minimum inhibitory concentration in Staphylococcus aureus infections: a systematic review and meta-analysis. Clin Infect Dis 54:755-771

4. Holmes NE, Turnidge JD, Munckhof WJ et al (2011) Antibiotic choice may not explain poorer outcomes in patients with Staphylococcus aureus bacteremia and high vancomycin minimum inhibitory concentrations. J Infect Dis 204(3):340-347

5. Cataldo MA, Tacconelli E, Grilli E, Pea F, Petrosillo N (2012) Continuous versus intermittent infusion of vancomycin for the treatment of Grampositive infections: systematic review and meta-analysis. J Antimicrob Chemother 67(1):17-24

6. Liu C, Bayer A, Cosgrove SE et al (2011) Clinical practice guidelines by the Infectious Diseases Society of America for the treatment of methicillinresistant Staphylococcus aureus infections in adults and children. Clin Infect Dis 52(3):e18-e55

7. Wunderink RG, Niederman MS, Kollef MH et al (2012) Linezolid in methicillin-resistant Staphylococcus aureus nosocomial pneumonia: a randomized, controlled study. Clin Infect Dis 54(5):621-629

8. Jiang H, Tang RN, Wang J (2013) Linezolid versus vancomycin or teicoplanin for nosocomial pneumonia: meta-analysis of randomized controlled trials. Eur J Clin Microbiol Infect Dis 32(9):1121-1128

9. Murray KP, Zhao JJ, Davis SL et al (2013) Early use of daptomycin versus vancomycin for methicillin-resistant Staphylococcus aureus bacteremia with vancomycin minimum inhibitory concentration $>1 \mathrm{mg} / \mathrm{L}:$ a matched cohort study. Clin Infect Dis 56:1562-1569
10. Gould IM, Miró JM, Rybak MJ (2013) Daptomycin: the role of high-dose and combination therapy for Gram-positive infections. Int J Antimicrob Agents 42(3):202-210

11. Schweizer ML, Furuno JP, Harris AD et al (2011) Comparative effectiveness of nafcillin or cefazolin versus vancomycin in methicillin susceptible Staphylococcus aureus bacteremia. BMC Infect Dis 11:279

12. Holmes NE, Tong SYC, Davis JS, van Hal SJ (2015) Treatment of methicillinresistant Staphylococcus aureus: vancomycin and beyond. Semin Respir Crit Care Med 36:17-30

13. Montravers P, Dupont H, Bedos J-P et al (2014) Tigecycline use in critically ill patients: a multicentre prospective observational study in the intensive care setting. Intensive Care Med 40:988-997

14. Dilworth TJ, Ibrahim O, Hall P, Sliwinski J et al (2014) $\beta$-Lactams enhance vancomycin activity against methicillin-resistant Staphylococcus aureus bacteremia compared to vancomycin alone. Antimicrob Agents Chemother 58:102-109

15. Davis JS, Sud A, O'Sullivan MV, Robinson JO et al (2016) Combination of vancomycin and $\beta$-Lactam therapy for methicillin-resistant Staphylococcus aureus bacteremia: a pilot multicenter randomized controlled trial. Clin Infect Dis 62(2):173-180

16. Gillet $Y$, Dumitrescu O, Tristan A, Dauwalder O et al (2011) Pragmatic management of Panton-Valentine leukocidin-associated staphylococcal diseases. Int J Antimicrob Agents 38(6):457-464

17. François B, Chastre J, Eggiman P, Laterre PF et al (2016) The SAATELLITE and EVADE clinical studies within the COMBACTE consortium: a publicprivate collaborative effort in designing and performing clinical trials for novel antibacterial drugs to prevent nosocomial pneumonia. Clin Infect Dis 63(Suppl 2):S46-S51 